\title{
DADOS MULTI-SENSORES PARA RECONHECIMENTO E MAPEAMENTO DE AMBIENTES SEDIMENTARES NA PLANÍCIE DO RIO AMAZONAS
}

\author{
MULTI-SENSOR DATA TO RECOGNIZE AND MAP SEDIMENTARY ENVIRONMENTS IN THE \\ AMAZONAS RIVER LOWLAND
} \author{
Estadual de Campinas - UNICAMP. E-mail: simmonviegass@gmail.com \\ E-mail: suzanpequeno@unb.br

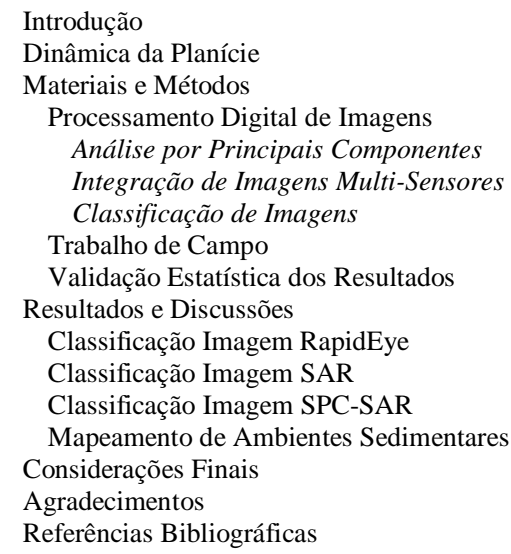

Simmon Viegas de SOUZA ${ }^{1}$, Suzan Waleska Pequeno RODRIGUES ${ }^{2}$

${ }^{1}$ Programa de Pós-Graduação em Geociências na linha de Geotecnologias Aplicadas ao Estudo de Recursos Naturais na Universidade

${ }^{2}$ Universidade de Brasília, Instituto de Geociências. Campus Universitário Darcy Ribeiro ICC - Ala Central. Brasília - DF.

RESUMO - Este trabalho propôs utilizar imagens de Synthetic Aperture Radar (SAR) que operam no intervalo das microondas, assim como imagens ópticas que operam no Visible-Near Infrared (VNIR) para reconhecer e mapear os principais ambientes fluviais do Baixo Amazonas. A área-teste (Ilha Grande do Tapará, que correspondente ao setor central do Baixo Rio Amazonas) foi escolhida com o intuito de aumentar a acurácia e qualidade de pontos de controle usados na interpretação dos ambientes da planície. O mapeamento das feições sedimentares foi baseado em classificações não supervisionadas e intepretação visual das imagens Ópticas, SAR e da imagem SPC-SAR resultante do sinergismo entre as imagens iniciais. Os resultados permitiram a identificação de classes lama, areia, vegetação arbórea e pasto aquático, resultando na associação de ambientes de lagos, diques, canais fluviais, deltas fluviais e praia fluvial. Os índices de acurácia geral obtidos pelos classificadores foram de 93,33\% e 0,90 para a imagem do sensor óptico; 93,33\% e 0,89 para a imagem SAR; e 78,25\% e 0,63 para o produto SPC-SAR. Os resultados indicam que o uso dos dados associados nos fornece importantes e precisas informações sobre a planície e, ainda, que o produto integrado contribuiu para o mapeamento da área estudada. Palavras-chave: Rio Amazonas, Ambientes Sedimentares, Sensoriamento Remoto, Sinergismo.

\begin{abstract}
This work proposes the use of Synthetic Aperture Radar (SAR) images that operate in the microwave range, as well as optical images that operate in Visible-Near Infrared (VNIR) to recognize and map the main river environments of the Lower Amazon. The test area (Ilha Grande do Tapará, corresponding to the central sector of the Lower Amazon River) was chosen with the purpose of increasing the accuracy and quality of control points used in the interpretation of the lowland environments. The mapping of the sedimentary features was based on unsupervised classifications and visual interpretation of the optical images, SAR and the SPC-SAR image resulting from the synergism between the initial images. The results allowed the identification of mud, sand, tree vegetation and aquatic pastures, resulting in the association of environments of lakes, dikes, fluvial channels, river deltas and fluvial beach. The general accuracy indices obtained by the classifiers were $93.33 \%$ and 0.90 for the optical sensor image; $93.33 \%$ and 0.89 for the SAR image; and $78.25 \%$ and 0.63 for the SPC-SAR product. The results indicate that the use of the associated data provides us with important and accurate information about the plain and also that the integrated product contributed to the mapping of the studied area.

Keywords: Amazonas River, Environment Sedimentary, Remote Sensing, Synergism.
\end{abstract}

\section{INTRODUÇÃO}

O Rio Amazonas possui um dos maiores e mais complexos sistemas fluviais do mundo (Pacheco \& Brandão, 2012), dividido tradicionalmente, devido sua extensão, em quatro regiões: o Alto Solimões, o Médio Amazonas, o Baixo Amazonas e a Região Marajoara, além de ser considerado um dos maiores rios que já foram identificados no mundo (Ab'Sáber, 2010).
O Baixo Amazonas, foco deste estudo, é dominado pela planície de inundação como foi estimado pelo Projeto Radar da Amazônia (RADAM), a partir de mapas geomorfológicos (Araújo et al., 1976).

Ao longo dos anos, boa parte das pesquisas envolvendo a região têm sido em relação a produção de dados sobre espécies de vegetação e 
áreas inundáveis, porém, ainda há um déficit no mapeamento geológico e cartográfico.

$\mathrm{Na}$ região do Baixo Amazonas, planície amazônica, são escassos os estudos e conjunto de informações que dizem respeito a localização, extensão, além de outras características básicas dos corpos d'água e de áreas alagáveis da planície (Lehner \& Doll, 2004).

A extensa dimensão, a dinâmica sazonal e, principalmente, a dificuldade de acesso limitam a extração de informações dessa região, o que torna o sensoriamento remoto a forma mais viável para fazer caracterização e monitoramento desse ambiente (Novo et al., 1998).

Os dados de sensoriamento favorecem a obtenção de informações atualizadas sobre diversas e extensas áreas da superfície terrestre, além da crescente qualidade das propriedades dos dados, o que possibilita o monitoramento mais preciso do espaço-tempo destas áreas (Lehner \& Doll, 2004).

Os dados produzidos e fornecidos por agências de pesquisa nos permitem levantar informações geográficas relevantes que possibilitam analisar as variações na planície no domínio do espaço e do tempo. Uma das maiores dificuldades em mapear o norte do país, principalmente a Amazônia, por sensoriamento remoto, é a grande quantidade de nuvens que aparecem nos dados de sensores ópticos e, por isso, torna-se inevitável que alguns setores não apresentem informações da superfície.

Com o surgimento e disponibilização de dados de microondas o problema das nuvens foi consideravelmente resolvido, otimizando a identificação de feições da superfície, pois os sensores que atuam no intervalo das microondas (e.g. Synthetic Aperture Radar) têm capacidade de penetração por conta dos maiores comprimentos de onda (Gonçalves et al., 2009). As grandes vantagens dos sensores de radar de abertura sintética ou Synthetic Aperture Radar (SAR) são que não são limitados por cobertura de nuvens ou iluminação solar e são capazes de detectar inundação abaixo da vegetação em alguns comprimentos de onda (Melack et al., 1994). Entretanto, o sinal de radar é sensível a rugosidade da superfície, o que pode causar confusão na superfície de corpos d'água que nem sempre são lisos (Bryan, 1981).

A definição de fusão de dados de sensores remotos é um procedimento em que são utilizados métodos e ferramentas para a fusão de dados originados de diferentes fontes, com o objetivo de explorar a integração desses dados para gerar mais informações e com maior qualidade que não poderiam ser extraídas dos dados isolados (Henderson \& Lewis, 1998; Paradella et al., 2000).

Existem diversas técnicas de fusão para realizar a integração de dados de sensoriamento remoto.

Na literatura (Harris et al., 1994; Wald, 1998; Pohl, 1998) vários trabalhos descrevem estes métodos através da transformação IHS (Inntensity, Hue, Saturation), que é a transformação dos componentes vermelho (Red), verde (Green) e azul (Blue) nos componentes de intensidade (I), matiz (H) e saturação (S), que pode ser utilizada para geração de composições coloridas reduzindo a correlação interbanda, além de combinar imagens de sensores diferentes (Teixeira, 2006).

Teixeira (2006) mostra que os tripletes de bandas foram testados em bandas TM (Thematic Mapper) para a integração com imagem SAR, e uma das combinações testadas foi o uso das três primeiras componentes obtidas a partir da análise do principal componente.

A possibilidade de gerar informações complementares nas diferentes faixas do espectro eletromagnético (microondas, infravermelho e visível), obtidas com o produto da fusão das diferentes imagens, indica grandes avanços nos estudos a partir do sensoriamento remoto de áreas pouco estudadas justamente pelas dificuldades causadas pelas interferências atmosféricas.

Os resultados dessa abordagem metodológica têm como resultado o produto híbrido, com maior qualidade e mais informações para extrações dos atributos, além de maior capacidade de identificação dos ambientes (Gonçalves et al., 2009).

Neste contexto, o objetivo deste trabalho foi integrar informações de dois importantes dados obtidos por sensoriamento remoto e que atuam em diferentes comprimentos de onda (Ópticos: VNIR e SAR: microondas) (e.g. Rodrigues \& Souza Filho, 2011), para reconhecer os principais componentes da planície do Rio Amazona e associá-los a feições sedimentares de origem fluvial.

A área de estudo escolhida para a realização deste trabalho é denominada Ilha Grande do Tapará, está localizada na região norte do Brasil, porção oeste do Estado do Pará, inserida na mesorregião do Baixo amazonas e microrregião de Santarém e compõe a planície fluvial do Rio 
Amazonas. A Ilha Grande do Tapará (Figura 1) fica distante a $24 \mathrm{~km}$ da sede da cidade de Santarém, está localizada dentro da bacia do Amazonas, sendo dominada pelas águas do Rio Amazonas, além de ser caracterizada por lagos em forma de canais e por cobertura vegetal de várzea dominante.

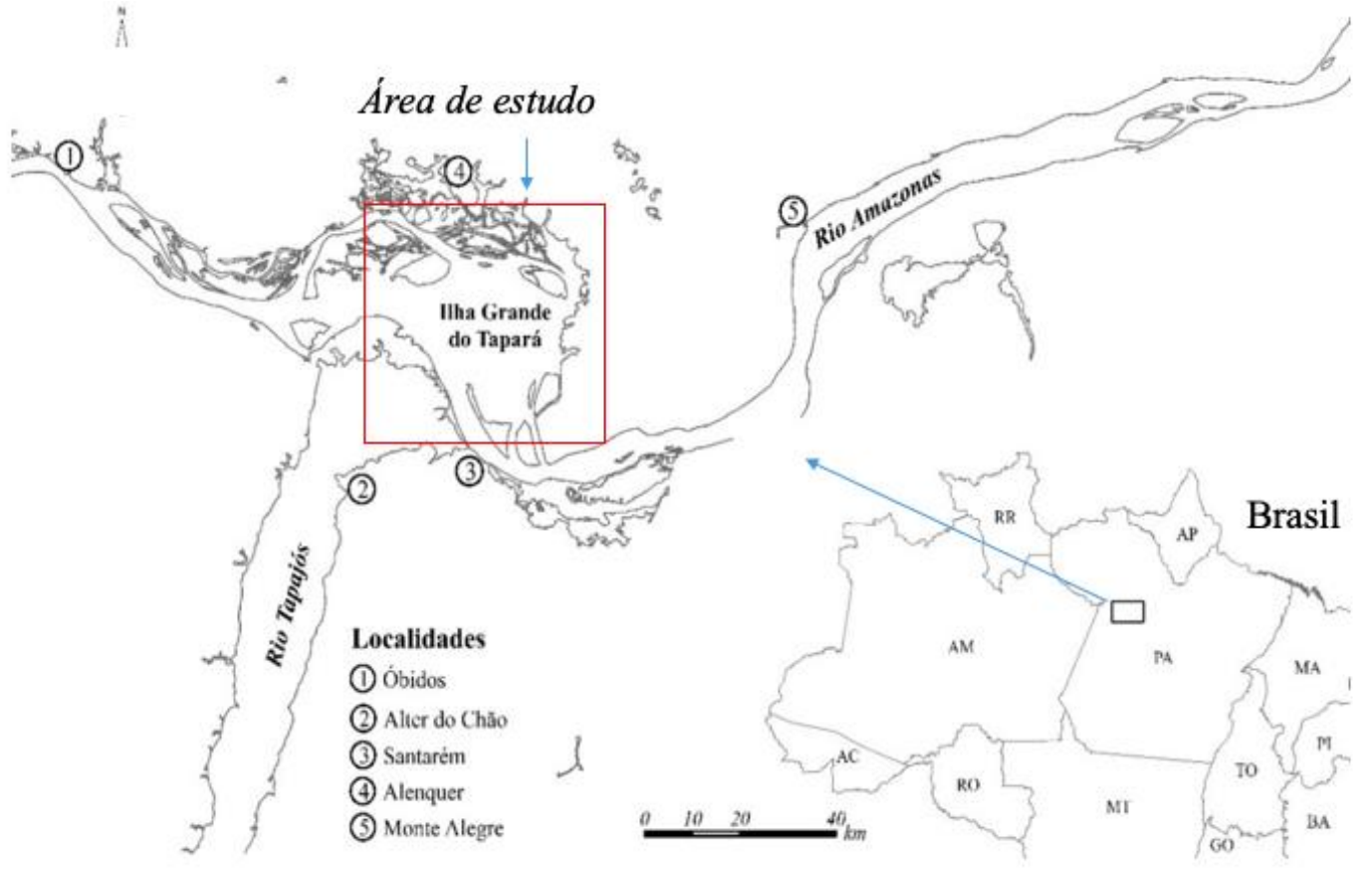

Figura 1 - Mapa de localização da Planície do Baixo Amazonas onde está inserida a Ilha Grande do Tapará.

\section{DINÂMICA DA PLANÍCIE AMAZÔNICA}

$\mathrm{Na}$ folha de Santarém (SA.21) foram identificadas oito unidades geomorfológicas (Araújo et al., 1976), e dentre estas oito, a área de estudo (Ilha Grande do Tapará) corresponde ao que Araújo et al. (1976) identificou como sendo a planície amazônica que se mantém no eixo principal no Rio Amazonas e sofre influência do Alto Estrutural de Monte Alegre, o que proporcionou as diferenças na forma do relevo da região (Cunha et al., 1994). As principais características da Planície Amazônica são as cheias e vazantes, que são causadas pelo pulso de inundação do Rio Amazonas, o que permitiu a subdivisão da planície em áreas alagadas e áreas inundáveis. As planícies de inundação ou aluviais são geradas a partir de processos físicosgeológicos-geomorfológicos que são resultados do fluxo hidrodinâmico e que produzem grandes variedades de sub-ambientes sedimentares com as mais variadas formas (Wittmann et al., 2004). Nas planícies de inundação estão registradas mudanças histórico-geológicas do ambiente em que a planície e seus sub-ambientes foram formados (Lewin, 1996). Seus sedimentos constituídos por conglo- merados, areias, siltes e argilas, que são os aluviões antigos e os mais novos, que predominam em todos os rios da região. Os aluviões mais "velhos" formam os terraços antigos e são relacionados a topografias mais elevadas estando discordantemente sobre as unidades mais antigas da região, que estão presentes nos vales dos rios Tapajós, Jatapú e Nhamundá.

Por outro lado, os aluviões recentes são quase sempre inconsolidados e se distribuem ao longo das calhas dos rios, igarapés e demais cursos d'água, formando terraços aluvionares mais recentes (Silva et al., 2011). Estes aluviões representam a grande fisiografia da bacia Amazônica, ou seja, a planície de inundação do Rio Amazonas, e pertencem a ela as áreas denominadas de alagadas e inundáveis, as quais estão submetidas diretamente ao controle do Rio Amazonas. A Planície Fluvial Alagada corresponde às áreas que permanecem submersas mesmo durante o período de menor volume do rio. Já a Planície Fluvial Inundável é caracterizada por áreas que são alagadas apenas no período de maior volume do rio (Silva et al., 2011).

\section{MATERIAIS E MÉTODOS}

Os dados utilizados neste trabalho estão contidos na tabela 1. As imagens do sensor REIS do satélite RapidEye foram adquiridas a partir de GeoCatálogo do Ministério do Meio Ambiente 
para fins de pesquisa científica e as imagens SAR-SIPAM foram obtidas na missão do Sistema de Vigilância da Amazônia (SIVAM) e também foram cedidas para fins de pesquisa pelo Sistema de Proteção da Amazônia (SIPAM).

O RapidEye é um sistema composto por cinco satélites idênticos localizados na mesma orbita e igualmente espaçados, que foram lançados em agosto de 2008 e que apresentam como uma das suas principais características a resolução temporal de 5,5 dias.

O sensor a bordo dos satélites é multiespectral, chamado de RapidEye Earth Imaging System (REIS). Esse sensor apresenta como peculiaridade a banda espectral na faixa do RED-EDGE. Essa banda foi desenvolvida para a discriminação de vegetação, situada na faixa do espectro eletromagnético que tem correlação com a fluorescência da clorofila. Portanto, esta banda pode ser utilizada para discriminar diferentes espécies de vegetação e avaliar a ocorrência de processos de eutrofização em reservatórios. A tabela 1 mostra as principais características do sensor REIS.

A missão SAR-SIPAM foi realizada pelo Sistema de Proteção da Amazônia (SIPAM) em parceria com o Ministério do Meio Ambiente (MMA). O sistema SAR-SIPAM opera na banda L $(1,27 \mathrm{GHz}$ de frequência e $23,9 \mathrm{~cm}$ de comprimento de onda), com dados polarimétricos que permitem o envio e recebimento de radiação eletromagnética nas polarizações vertical, horizontal e cruzada (HH, VV, HV e VV), além da banda $X$ (frequência de 9,69 GHz e comprimento de onda de aproximadamente $3,1 \mathrm{~cm}$ ) com apenas uma polarização HH. Devido à baixa alteração/modificação das feições analisadas na superfície da área estudada não houve prejuízo em relação as diferentes datas entre as imagens do sensor óptico e micro-ondas adotados. As etapas para execução do trabalho estão representadas no fluxograma da figura 2.

Tabela 1 - Características das imagens utilizadas.

\begin{tabular}{c|c|c|c|c}
\hline Plataforma & Sensor & Bandas & Resolução espacial & Data de aquisição \\
\hline RapidEye & REIS & $\begin{array}{c}1(440-510 \mathrm{~nm}), 2(520-590 \mathrm{~nm}), \\
3(630-685 \mathrm{~nm}), 4(690-730 \mathrm{~nm}), \\
5(760-850 \mathrm{~nm})\end{array}$ & 6,5 metros & $18 / 10 / 2013$ \\
\hline SAR-SIPAM & SAR & $\mathrm{HH}, \mathrm{HV}, \mathrm{VH}$ & 18 metros & 2008 \\
\hline
\end{tabular}

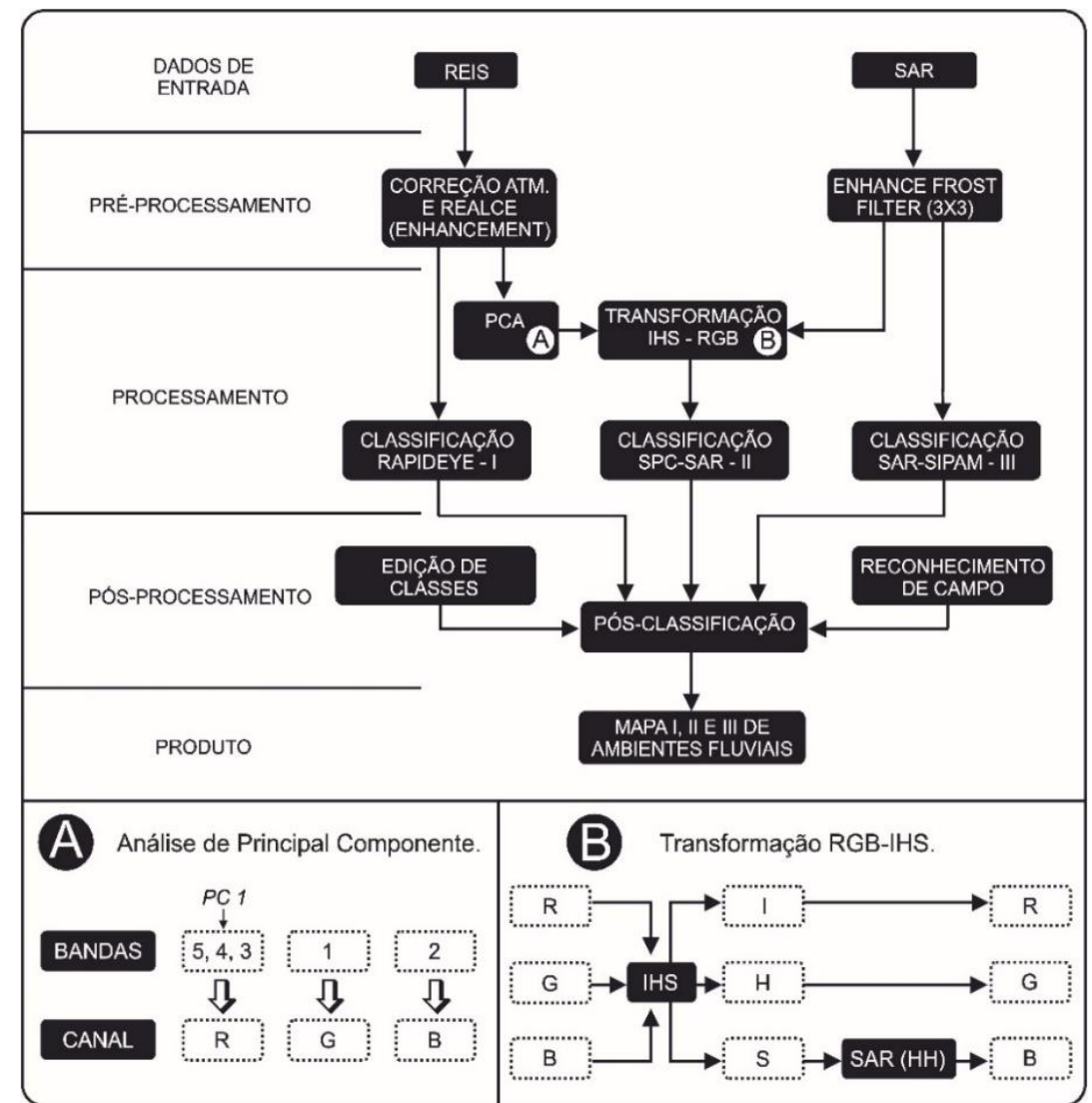

Figura 2 - Fluxograma ilustrativo do fluxo de trabalho utilizado para correção, processamento e resultados esperados (Adaptado de Rodrigues \& Souza Filho, 2011). 


\section{Processamento de Imagens}

$\mathrm{Na}$ etapa de pré-processamento de imagens as bandas 1, 2, 3, 4 e 5 do sensor REIS/RapidEye passaram por correção atmosférica, de acordo com o método de Chavez (1988), para a subtração dos pixels escuros e melhor visualização das feições a serem estudadas.

Após esta etapa, as imagens foram realçadas com a aplicação do efeito de contraste linear. Pelo fato de que a área estudada possui grande extensão e, por isso, é recoberta por mais de uma cena, houve a necessidade de mosaicar duas cenas adjacentes lateralmente para cobrir toda a área.

$\mathrm{Na}$ imagem SAR foi aplicado o filtro FEFROST $3 \times 3$, para suavização dos ruídos (speckle). Ambas as imagens foram recortadas para melhor delimitação da área de estudo. Estes processamentos foram realizados no software PCI Geomatics v. 2015 a partir de sua biblioteca de algoritmos.

\section{Análise por Principais Componentes}

O procedimento chamado de Análise por Principal Componente é baseado na utilização das bandas que possuem relações espectrais ente si, servindo como dados de entrada. A partir disso, as imagens agrupadas seletivamente têm por objetivo mapear a maior parte das informações (variância) no primeiro componente (PC1) pela correlação elevada entre as bandas usadas. Neste trabalho foram selecionadas as bandas 3, 4 e 5 do sensor REIS para a realização da APC.

$\mathrm{O}$ produto da análise por componente principal foram os PC's que mantém as principais informações das bandas utilizadas nesta técnica. Para a aplicação desta técnica foi utilizada a função Principal Component Analysis (PCA) disponível no ArcToolbox do software ArcGIS v. 10.4.

\section{Integração de Imagens Multi-Sensores}

Para a integração dos dados multisensores, foi usado o método de fusão de dados, chamado de Selective Principal Component Analysis - SPC. A transformação IHS-RGB foi realizada através do ArcToolbox presente no software ArcGIS, tendo como dados de entrada o PC1 (resultante da análise de principal componente) e as bandas 1 e 2 da imagem REIS.

Em seguida, na transformação inversa, a componente saturação (S) foi substituída pela banda $\mathrm{HH}$ do SAR-SIPAM, mantendo-se as componentes I e H. Antes da integração dos dados REIS-SAR, as duas imagens foram configuradas para o mesmo sistema de projeção, mesmo DATUM e mesmo tamanho dos pixels (15 metros). Assim, o produto gerado a partir da integração foi o SPC-SAR.

\section{Classificação de Imagens}

A classificação automática de imagens nada mais é que o agrupamento de pixels com mesma ou parecida resposta espectral. Desta forma, são criadas áreas que correspondem ao tipo de cobertura da superfície terrestre que foi imageada.

Áreas como água, vegetação, solo, etc., e que são chamadas de classes. Neste trabalho foram feitas classificações não supervisionadas, onde cada pixel da imagem é associado a uma resposta espectral sem que o usuário tenha identificado previamente as classes a serem geradas.

Os três produtos de sensoriamento remoto usados neste trabalho, imagem RapidEye (composição R1G2B3), imagem SAR-SIPAM (composição RHHGHVBVH) e o SPC-SAR, foram classificados pelo algoritmo ISOSEG, que faz agrupamentos ou "clustering", aplicado sobre um determinado conjunto de regiões, caracterizadas por seus atributos estatísticos de média, matriz de covariância e área, que está presente no software PCI Geomatics 15.

\section{Trabalho de Campo}

Foram realizados levantamentos de campo no mês de outubro de 2016, em que foi efetivada a verdade-terreno com coleta de pontos de controle utilizando GPS (Global Positioning System) de navegação, caminhamentos (tracking) e check de campo das unidades morfológicas e geológicas da área.

O objetivo do trabalho de campo foi fazer a validação dos ambientes fluviais que anteriormente haviam sido reconhecidos nas imagens dos sensores remotos.

\section{Validação Estatística}

A validação para os produtos das três classificações foi gerada a partir do índice Kappa, sendo feita uma amostragem de 400 pixels para cada classificação, em modo random, seguindo a metodologia de Cohen (1960), tendo como base um mosaico de imagens de alta resolução espacial $(50 \mathrm{~cm})$ do satélite WorldView-2, mosaico este que foi obtido na plataforma GoogleEarth.

Além disso, foi realizado o cálculo das matrizes de confusão, que mostram o percentual de pixels que foram mapeados de forma correta. 
Ainda foi usado o Índice Kappa (Tabela 2) como parâmetro de avaliação da concordância entre verdade terrestre e os mapas temáticos gerados pelas classificações. Foram realizados também os cálculos dos erros de comissão e omissão.

A comissão é quando um pixel é classificado como sendo de uma classe " $X$ " quando, na verdade, pertence a uma classe "Y". A omissão ocorre quando um pixel que pertence a uma classe " $X$ " é classificado como pertencente a uma outra classe qualquer (Jensen 1996).
Tabela 2 - Coeficiente Kappa e os conceitos associados a cada coeficiente (Cohen, 1960).

\begin{tabular}{c|c}
\hline Coeficiente Kappa & Desempenho da classificação \\
\hline$<0$ & Péssimo \\
\hline $0<\mathrm{K} \leq 0,2$ & Mau \\
\hline $0,2<\mathrm{K} \leq 0,4$ & Razoável \\
\hline $0,4<\mathrm{K} \leq 0,6$ & Bom \\
\hline $0,6<\mathrm{K} \leq 0,8$ & Muito Bom \\
\hline $0,8<\mathrm{K} \leq 1,0$ & Excelente \\
\hline
\end{tabular}

\section{RESULTADOS E DISCUSSÕES}

Os resultados das classificações não supervisionadas realizados nas imagens REIS/RapidEye, SAR-SIPAM e SPC-SAR, bem como os resultados das validações estatísticas realizadas para cada imagem serão apresentadas a seguir.

Para confirmar a acurácia das classificações foram analisadas as matrizes de confusão junto ao índice Kappa, além dos erros de comissão e omissão de cada classificação.

\section{Classificação Imagem RapidEye}

A partir do processamento da imagem digital do sensor REIS/RapidEye (Figura 3.A) de composição (R)1(G)2(B)3, foi gerada a classificação não supervisionada resultando no mapa da Figura 3.B, com 5 classes, sendo estas: lama, areia, vegetação alta, vegetação baixa e água. A partir da associação de classes e da interpretação das imagens foi possível a identificação na área de um leque de espraiamento que desemboca no interior da ilha, caracterizado por vegetação semiaquática e lama (Figura 3.B.1).

De acordo com o índice Kappa gerado para avaliar esta classificação, foram obtidos valores maiores que 0,71 (Classe Lama) de acurácia para as classes a partir dos 400 pixels amostrados na imagem, identificando quais foram comissionados, omitidos e corretamente classificados neste processamento (Tabela 3).

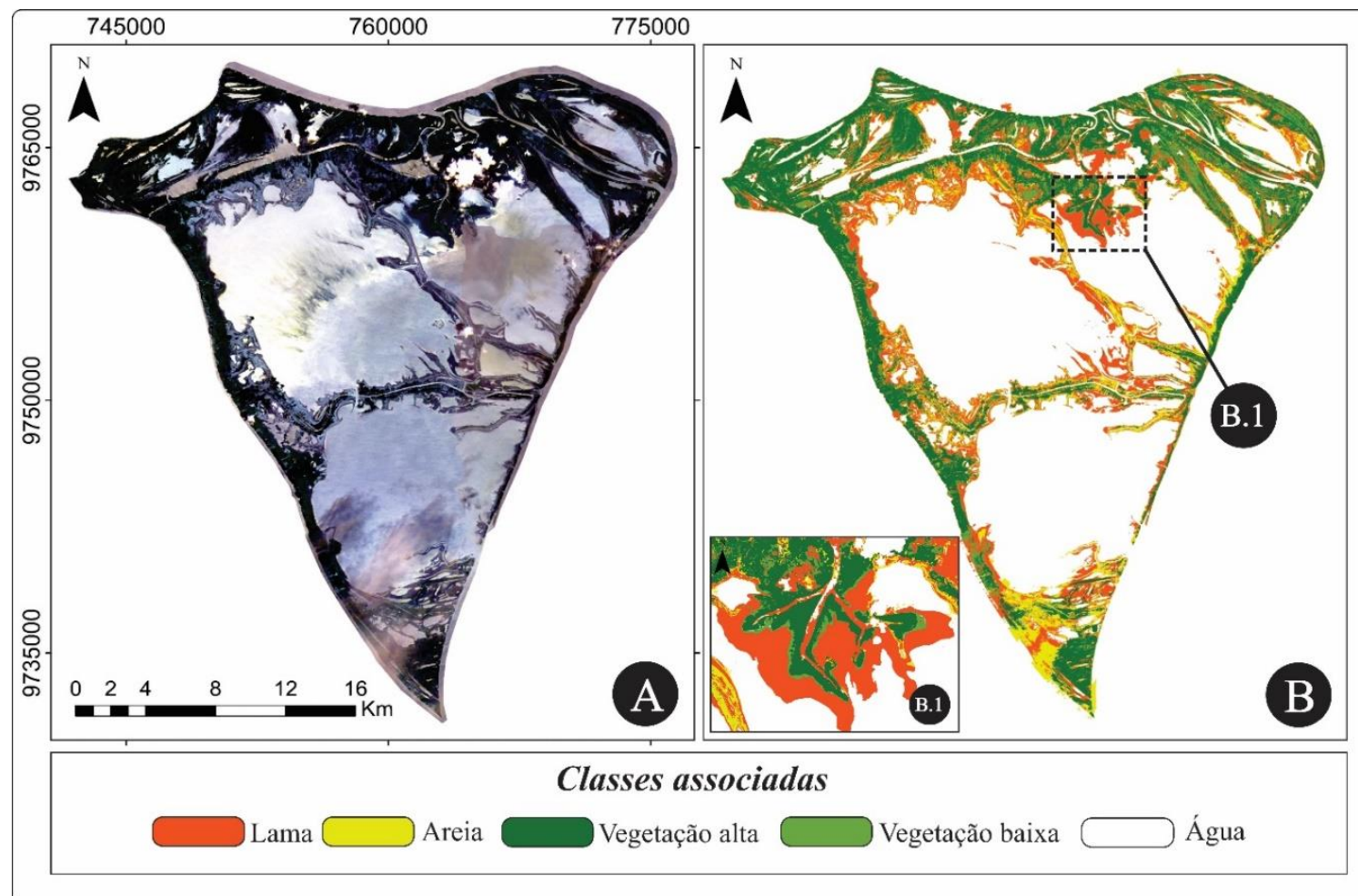

Figura 3 - A) Imagem RapidEye de composição (R)1(G)2(B)3; B) Resultado da classificação não supervisionada dos ambientes sedimentares; B.1) Detalhe do delta fluvial associado a altas cargas de sedimentos como areia e lama. 
Tabela 3 - Matriz de confusão da classificação não supervisionada ISOSEG baseada na imagem RapidEye com composição $(\mathrm{R}) 1(\mathrm{G}) 2(\mathrm{~B}) 3$.

\begin{tabular}{|c|c|c|c|c|c|c|c|c|c|}
\hline Classes & A & B & C & D & $\mathbf{E}$ & $\mathbf{F}$ & Total & $\begin{array}{c}\text { Erro } \\
\text { Usuário (\%) }\end{array}$ & \begin{tabular}{|c} 
Erro \\
Comissão (\%)
\end{tabular} \\
\hline $\mathbf{A}$ & 22 & 6 & 0 & 2 & 0 & 0 & 30 & 73,33 & 26,67 \\
\hline B & 2 & 11 & 0 & 0 & 0 & 0 & 13 & 84,61 & 15,39 \\
\hline $\mathbf{C}$ & 0 & 0 & 7 & 1 & 0 & 0 & 8 & 87,50 & 12,50 \\
\hline D & 4 & 0 & 1 & 29 & $\mathbf{0}$ & 0 & 34 & 85,29 & 14,71 \\
\hline $\mathbf{E}$ & 0 & 0 & 0 & 0 & 1 & 0 & 1 & 100,00 & 0,00 \\
\hline $\mathbf{F}$ & 0 & 1 & 0 & 0 & 0 & 313 & 314 & 99,68 & 0,32 \\
\hline Total & 28 & 18 & 8 & 32 & & 313 & 400 & & \\
\hline Erro Produtor (\%) & 78,57 & 61,11 & 87,50 & 90,62 & 100,00 & 100 & & & \\
\hline Erro Omissão (\%) & 21,43 & 38,89 & 12,50 & 9,38 & 0,00 & 0,00 & & & \\
\hline Kappa individual & 0,71 & 0,83 & 0,87 & 0,84 & 1,00 & 0,98 & & & \\
\hline Kappa Geral & $\mathbf{0 , 8 8}$ & & & \multicolumn{4}{|c|}{ Acurácia Geral = 95,75 } & & \\
\hline
\end{tabular}

Legenda - Lama: A; Areia: B; Vegetação Alta: C; Vegetação Baixa: D; Nuvem: E; No data: F.

Esta classificação obteve índices considerados Muito Bons a Excelentes (menor índice: 0,71; maior índice: 1,00).

Em relação as classes geradas para $o$ reconhecimento dos ambientes, a classe Vegetação Alta obteve comissão mais baixa $(12,50 \%)$ e a menor omissão $(9,38 \%)$ comparada as outras classes, indicando que esta teve uma boa classificação.

$\mathrm{O}$ valor "No data" inclui feições fora da área de interesse como água, além dos valores "Nuvens", onde ambos não entraram nesta discussão, mas foram computados de modo a validar toda a classificação.

O índice Kappa da classificação geral foi de
0,88, o que corresponde a uma classificação Excelente, segundo a tabela de conceitos de Cohen (1960).

\section{Classificação Imagem SAR}

$O$ processamento da imagem SAR-SIPAM (Figura 4.A) de composição (R)HH(G)HV(B)VH teve como resultado a classificação não supervisionada da figura 4.B, com 5 classes, sendo estas as mesmas classes geradas para a classificação da imagem REIS/RapidEye, exceto "Nuvem". Como pode ser observado nesta figura, a interpretação das imagens permitiu identificar diversas feições, tais como: lagos rodeados por brejos e cortados por diques fluviais, típicos do sistema fluvial, entre outros (Figura 4.B.1).

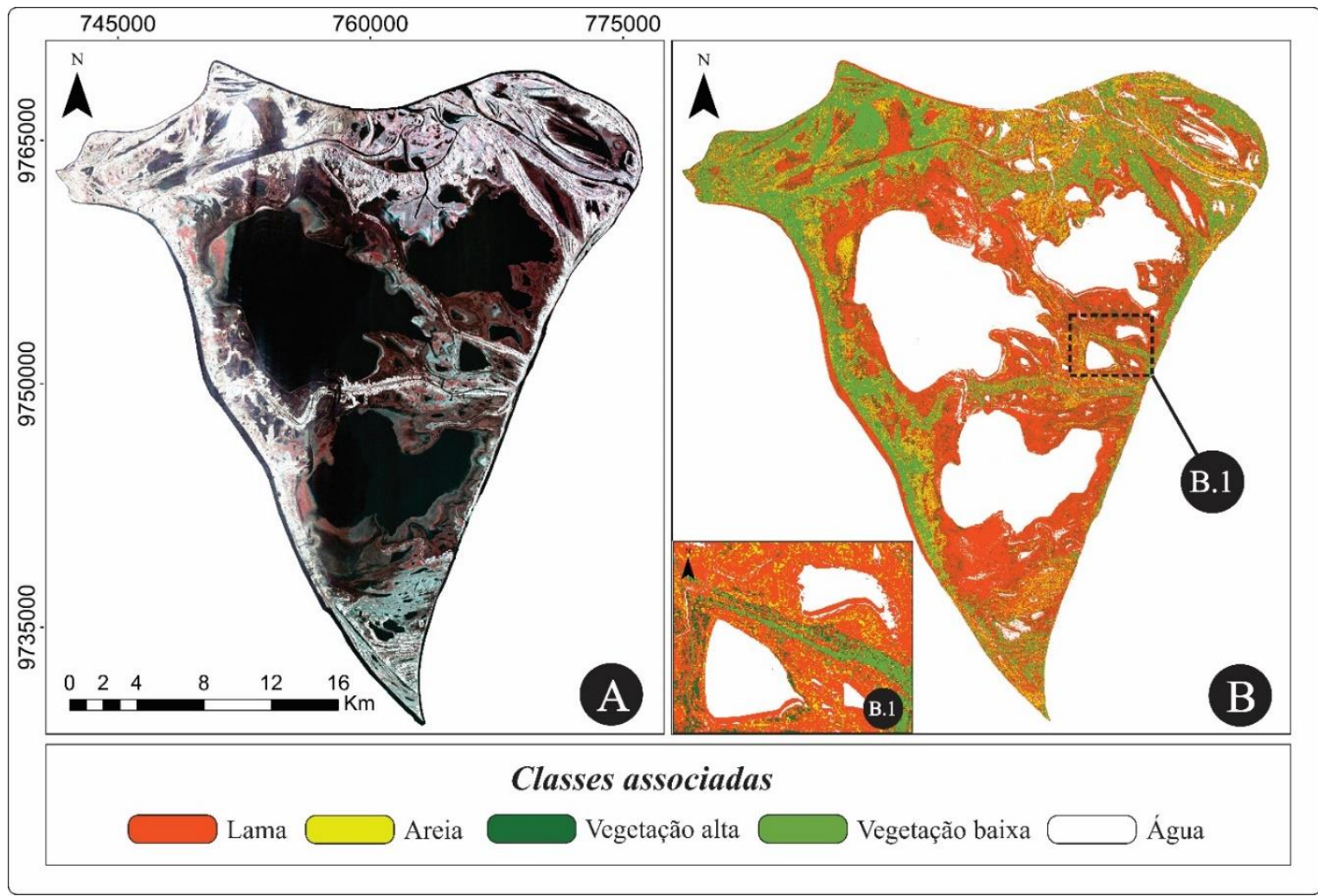

Figura 4 - A) Imagem SAR com composição RHH, GHV, BVH; B) Resultado da classificação não supervisionada dos ambientes sedimentares; B.1) Detalhe de lagos rodeados por brejos de lama e vegetação semiaquática. 
Os valores de índice Kappa gerados para as classes apontam índices que variam de Muito Bom a Excelente, fornecendo valores mínimos de 0,61 para a classe Areia e m 1,00 para a classe "No data" (Tabela 4). A partir dos 400 pixels amostrados para a validação, percebeuse uma boa classificação para as classes Vegetação Alta e Vegetação Baixa, enquanto que para a classe Areia houve 54,55\% de omissão para outras classes, principalmente para a classe Lama e 37,50,29\% de comissão, principal-mente para as classes Areia e Vegetação Baixa. Estes valores representam a proximidade das respostas de retorno do sinal do radar devido à similaridade destas feições na constituição do terreno.

Tabela 4 - Matriz de confusão da classificação não supervisionada ISOSEG baseada na imagem SAR com composição (R) $\mathrm{HH}(\mathrm{G}) \mathrm{HV}(\mathrm{B}) \mathrm{VH}$.

\begin{tabular}{|c|c|c|c|c|c|c|c|c|}
\hline Classes & $\mathbf{A}$ & B & $\mathbf{C}$ & D & $\mathbf{E}$ & Total & $\begin{array}{c}\text { Erro } \\
\text { Usuário }(\%)\end{array}$ & $\begin{array}{c}\text { Erro } \\
\text { Comissão }(\%)\end{array}$ \\
\hline $\mathbf{A}$ & 53 & 5 & 8 & 1 & 0 & 67 & 79,10 & 20,90 \\
\hline B & 2 & 5 & 1 & 0 & 0 & 8 & 62,50 & 37,50 \\
\hline $\mathbf{C}$ & 4 & 0 & 16 & 0 & 0 & 20 & 80,00 & 20,00 \\
\hline D & 0 & 1 & 5 & 28 & 0 & 34 & 82,35 & 17,65 \\
\hline $\mathbf{E}$ & 0 & 0 & 0 & 0 & 271 & 271 & 100,00 & 0,00 \\
\hline Total & 59 & 11 & 30 & 29 & 271 & 400 & & \\
\hline Erro do Produtor (\%) & 89,83 & 45,45 & 53,33 & 96,55 & 100,00 & & & \\
\hline Erro de Omissão (\%) & 10,17 & 54,55 & 46,67 & 3,45 & 0,00 & & & \\
\hline Kappa individual & 0,75 & 0,61 & 0,78 & 0,80 & 1,00 & & & \\
\hline \multicolumn{2}{|c|}{ Índice Kappa Geral =0,86 } & & & \multicolumn{3}{|c|}{ Acurácia Geral = 93,25 } & & \\
\hline
\end{tabular}

Legenda - Lama: A; Areia: B; Vegetação Baixa: C; Vegetação Alta: D; No data: E.

\section{Classificação Imagem SPC-SAR}

O produto SPC-SAR (Figura 5.A), foi classificado gerando o mapa da Figura 4.B, com 4 classes, sendo estas: lama, vegetação alta, vegetação baixa e água. A interpretação dos produtos permitiu identificar feições como os lagos em espira de meandro constituídos por vários espelhos d'água estreitos e paralelos dispostos em semicírculo (Figura 5.B.1) diques fluviais, canais e brejos.

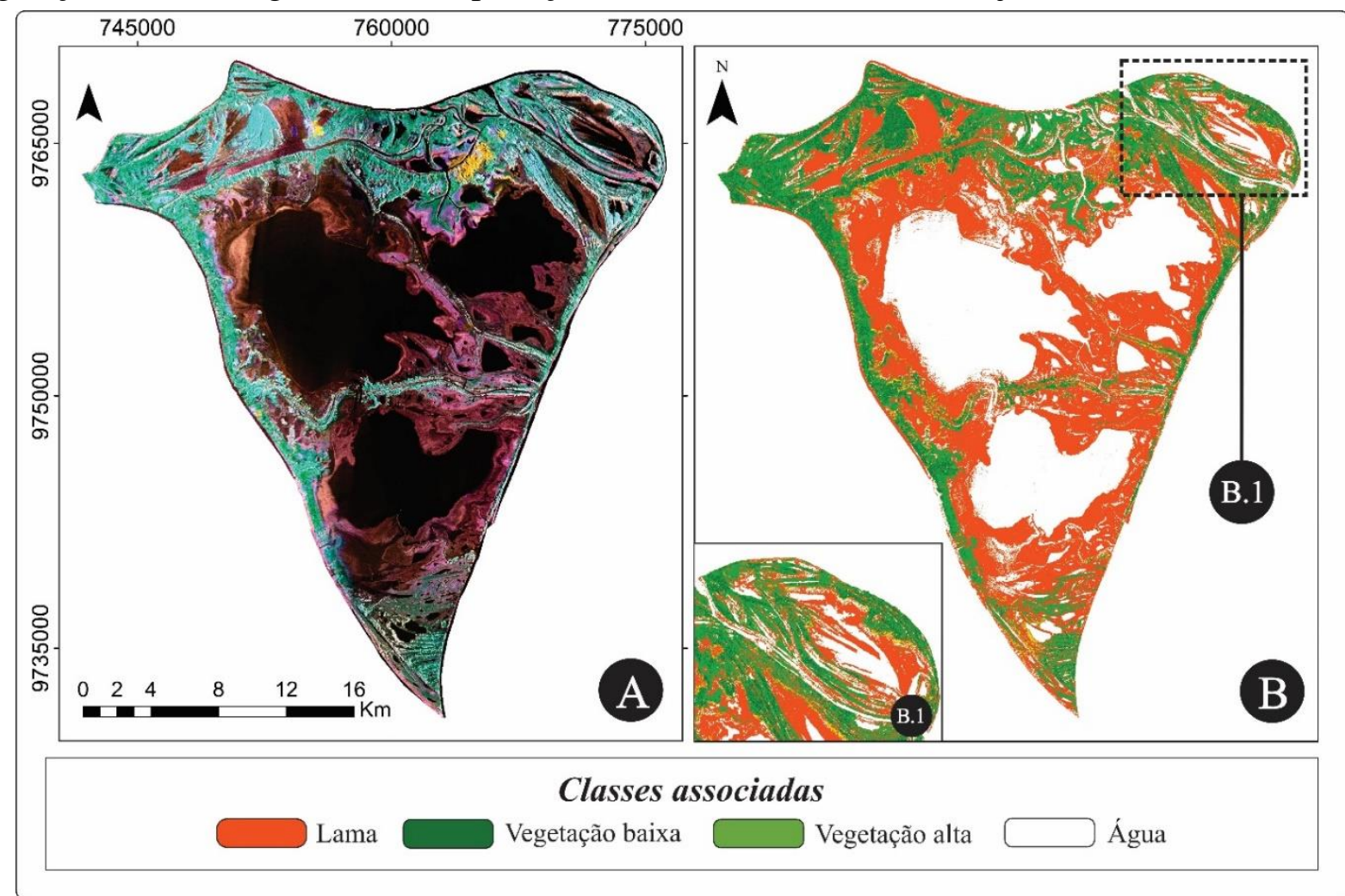

Figura 5 - A) Imagem SPC-SAR resultado da fusão de imagens de radar e óptica. B) Resultado da classificação não supervisionada do ambiente fluvial. B.1) Zoom detalhando a feição de lagos em espira de meandro comumente identificados na região amazônica. 
Neste produto os resultados foram satisfatórios, quando comparado aos produtos da classificação da imagem óptica e da imagem de microondas, mostrando com riqueza de detalhes as feições geomorfológicas do ambiente, o que é indicado pelos resultados dos dados de validação. A matriz de confusão da classificação do produto SPC-SAR indica que a imagem fusionada possui índice Kappa Muito $\operatorname{Bom}(0,62)$ quanto a classificação automática (Tabela 5). Dentre as classes geradas, que são determinantes para a classificação dos ambientes, a classe Vegetação Alta obteve a comissão mais baixa (10\%) e a menor omissão (10\%). Ou seja, este produto, nesta área, foi um bom delimitador e identificador de todas as classes necessárias para a identificação dos ambientes, assim como os produtos individuais.

Tabela 5 - Matriz de confusão da classificação não supervisionada ISOSEG baseada no produto SPC-SAR.

\begin{tabular}{|c|c|c|c|c|c|c|c|c|}
\hline Classes & $\mathbf{A}$ & B & $\mathbf{C}$ & D & $\mathbf{E}$ & Total & Erro Usuário (\%) & Erro Comissão (\%) \\
\hline $\mathbf{A}$ & 279 & 0 & 0 & 3 & 0 & 282 & 100,00 & 0,00 \\
\hline B & 1 & 24 & 0 & 5 & 0 & 30 & 80,00 & 20,00 \\
\hline $\mathbf{C}$ & 0 & 1 & 9 & 0 & 0 & 10 & 90,00 & 10,00 \\
\hline D & 0 & 5 & 1 & 63 & 5 & 74 & 85,13 & 14,87 \\
\hline $\mathbf{E}$ & 0 & 0 & 0 & 1 & 3 & 4 & 75,00 & 25,00 \\
\hline Total & 280 & 30 & 10 & 72 & 8 & 400 & & \\
\hline Erro Produtor (\%) & 76,42 & 80,00 & 90,00 & 87,50 & 37,50 & & & \\
\hline Erro Omissão (\%) & 23,58 & 20,00 & 10,00 & 12,50 & 62,50 & & & \\
\hline \multicolumn{9}{|l|}{ Kappa individual } \\
\hline \multicolumn{2}{|c|}{ Índice Kappa Geral = 0,629 } & & & \multicolumn{4}{|c|}{ Acurácia Geral $=78,25$} & \\
\hline
\end{tabular}

Legenda - No data: A; Vegetação Baixa: B; Vegetação Alta: C; Lama: D; Água: E.

\section{Mapeamento de Ambientes Sedimentares}

Com base na metodologia proposta para reconhecimento de ambientes na região do Baixo Amazonas, foram reconhecidos ambientes fluviais com dados de boa resolução espacial das imagens REIS/RapidEye e dados sem interferências atmosféricas das imagens SAR-
SIPAM, além da importante contribuição do SPC-SAR, produto integrado das imagens ópticas e radar.

Os resultados da aplicação destes dados de sensores nos mostram ser possível a caracterização das diferentes feições do sistema fluvial ao longo do Rio Amazonas (Figura 6).

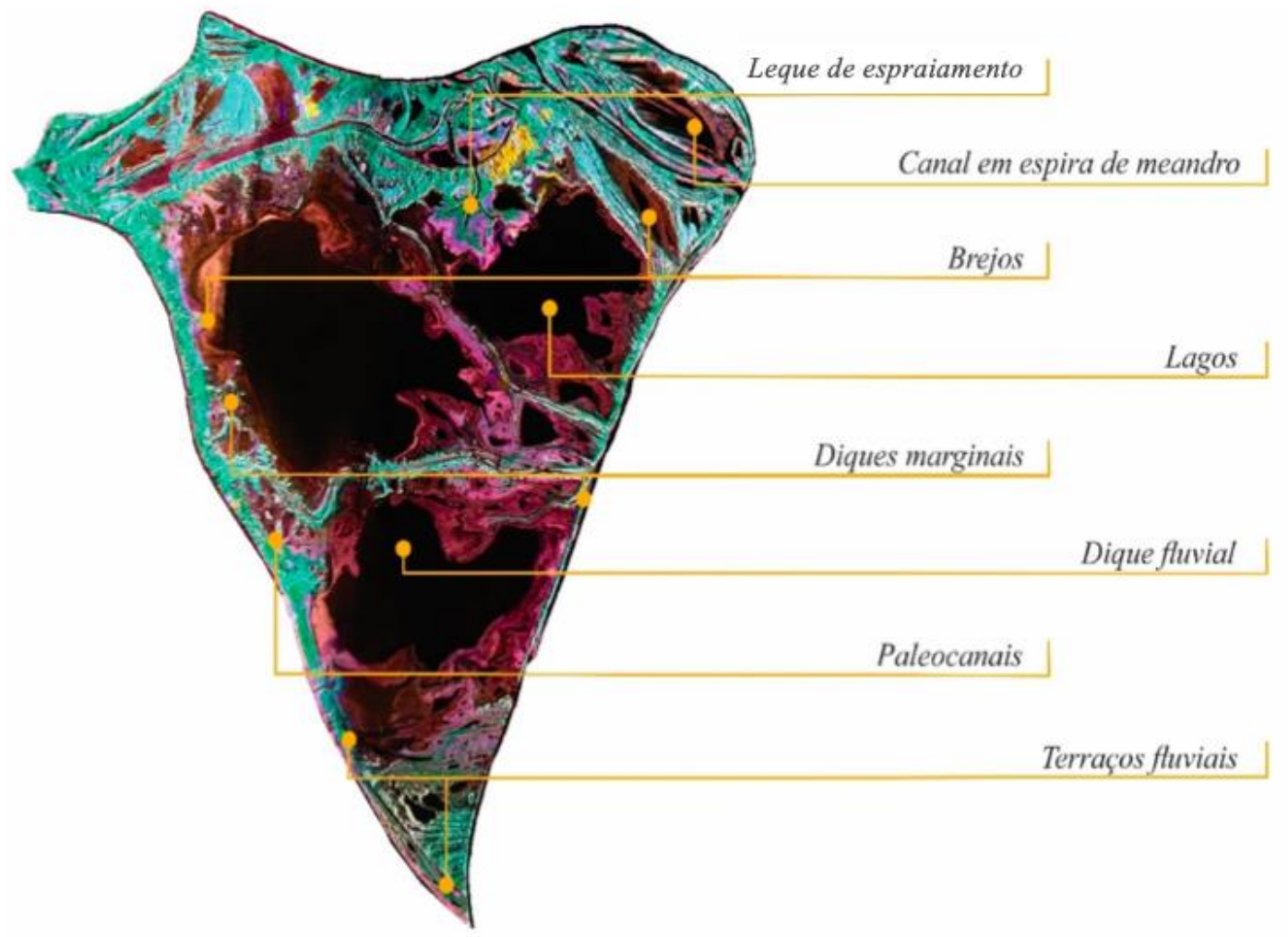

Figura 6 - Imagem SPC-SAR identificando os ambientes a partir da interpretação visual e classificação não supervisionada, associado ao trabalho de campo na área de estudo. 
Os processos de transporte e sedimentação que atuam constantemente na planície amazônica desenham as características particulares da região. Os sedimentos depositados na planície da Ilha Grande do Tapará podem ser considerados como originados de um único ambiente deposicional. No entanto, é notável que sua arquitetura sedimentar é variada formando diferentes sub-ambientes. Os lagos (Figuras 7A e 7B) da ilha geralmente são intermitentes, ligados diretamente ao ciclo de inundação Amazônica. São compostos principalmente por sedimentos mais finos por estarem fora dos canais que cortam a ilha.

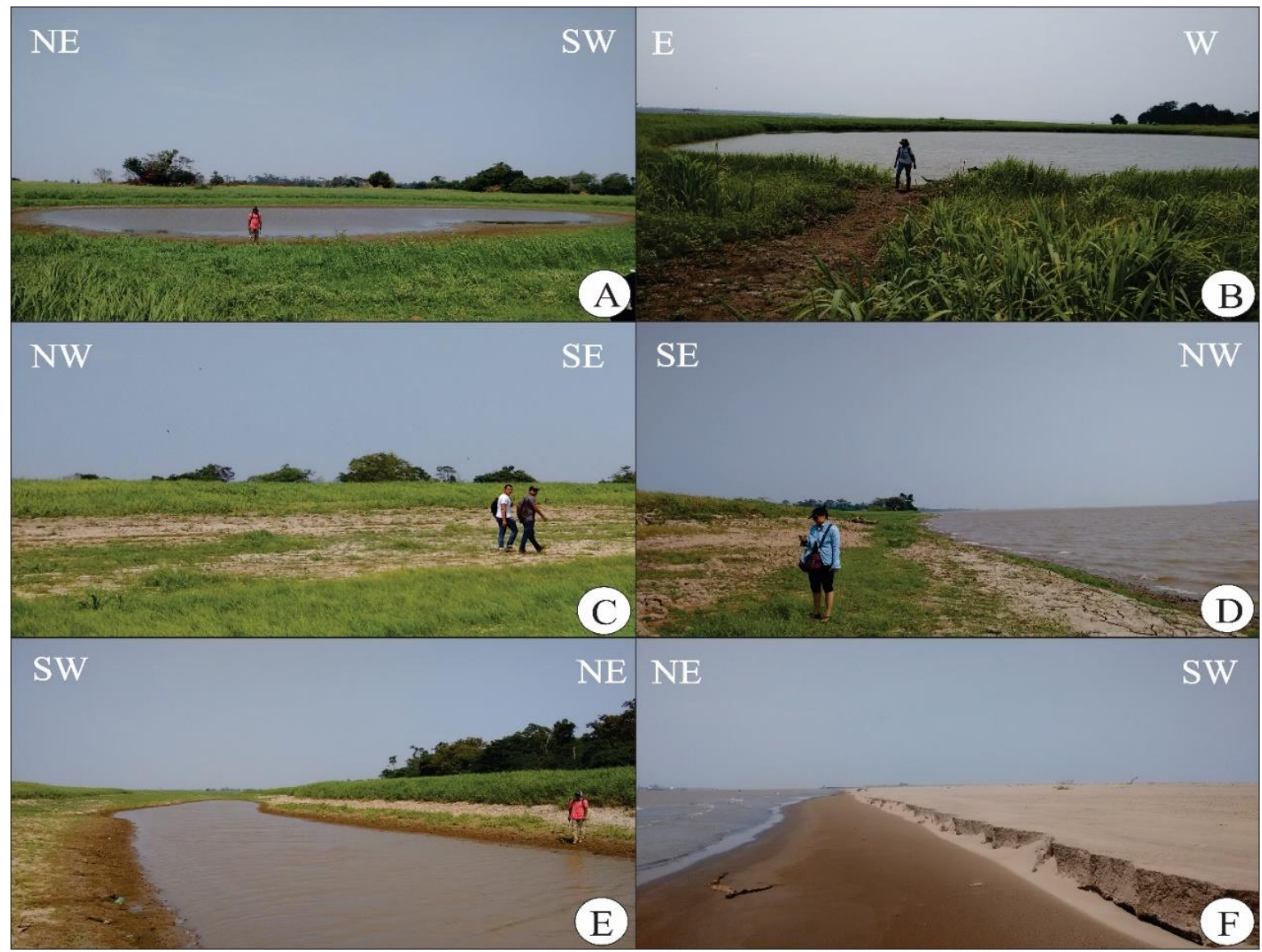

Figura 7 - A e B) são lagos temporários com presença de sedimentos finos e rodeados por vegetação de pasto natural; $\mathbf{C}$ e D) são áreas mais elevadas, associadas a terraços fluviais; E) representa os diques marginais; em F) um exemplo de ilha formada por acresção de sedimentos de granulometria fina e média, também identificadas como praias fluviais.

A estes sedimentos finos estão associados também matéria orgânica proveniente da própria vegetação da região. As praias fluviais são formadas às margens da ilha ou surgem na forma de barras em pontal nos canais que cortam a ilha e são controlados principalmente pelos processos de acresção lateral e vertical.

A formação dos leques de espraiamento (Figura 8) na região é associada ao transbordamento do rio que leva ao rompimento marginal dos canais, formando diques $e$, posteriormente, deltas internos que despejam altas cargas de sedimentos nos lagos da ilha. Os paleocanais são formados a partir da migração do canal principal ou podem ocorrer paleocanais intermitentes, ou seja, que surgem com a descida no nível do rio.

Possuem forma alongada e os limites das suas margens são bem definidos apresentando vegetações de médio porte, como pastos, em alguns casos. Estes lagos apresentam elevações seguidas de depressões, continuadamente, que ocorrem nas margens convexas dos meandros.

Cada elevação presente nestes lagos configura uma antiga margem do rio e as depressões são partes antigas no canal. Justamente nas depressões é que os lagos se formam, dando a forma de espira vista na imagem.

Brejos (campos inundados) são as áreas que estão entre as mais baixas da planície e permanecem a maior parte do tempo encharcadas. Nesses locais é comum a formação de lagos. Os brejos são formados quando as águas ultrapassam os diques naturais que estão nas margens dos canais fluviais.

A deposição nestes lagos ocorre por 
predominância do processo de acresção vertical, composta pela decantação dos materiais finos que foram transportados para dentro dos lagos.

Os diques marginais apresentam morfologia alongada e são formados nas margens da ilha quando a cheia ultrapassa os diques naturais por meio de canais abertos através deles formando canais para o interior da ilha. É importante ressaltar que, nas análises da imagem de radar, os diques se apresentam geralmente associados à vegetação arbórea e vegetação de pasto natural, como pode ser visto na figura 7.E.

Os terraços fluviais (Figura 7.C) são formados com o recuo do nível do rio, expondo a planície e criando espaço para o desenvolvimento de vegetação rasteira como gramíneas.

Nos terraços a maioria das geoformas da planície estão em forma de marcas, indicando quais os processos que ocorreram naquele local no momento em que a água cobria o terraço.

O sistema geomorfológico da Ilha Grande do Tapará foi desenvolvido com uma complexa morfologia de sistema fluvial. O conjunto de ambientes da área registram a evolução contínua da planície amazônica e permite o entendimento da sua dinâmica.

\section{CONSIDERAÇÕES FINAIS}

Como proposto nos objetivos deste trabalho, foram usadas imagens obtidas por sensores remotos, que contém diferentes características da superfície, com o intuito de realizar o reconhecimento de ambientes na planície amazônica, mais especificamente, na Ilha Grande do Tapará. A partir do processamento das imagens ópticas e de radar foi possível a identificação dos principais ambientes fluviais que compõem a área de estudo. As imagens obtidas por sensores ópticos forneceram informações relacionadas a características físicas e químicas dos objetos e as imagens de radar contribuíram com as informações morfológicas, incluindo altura, geometria e fornecendo subsídios para a individualização das vegetações, contribuindo significativamente para a identificação das feições da área.

Com o uso das imagens de sensoriamento remoto, foi possível a produção de mapas representando algumas das feições sedimentares amazônicas e, com isso, podemos concluir que as imagens dos sensores REIS/RapidEye e SARSIPAM tiveram excelentes resultados para a classificação automática, nos permitindo a identificação das classes de vegetação, lama e areia o que nos forneceu uma melhor precisão no reconhecimento das feições fluviais como: lagos, brejos, paleocanais, diques marginais, bancos de acresção, lagos em espira, delta fluvial e terraços. As classificações geraram bons valores de acurácia que variaram de Muito Bons a Excelentes, o que nos permite dizer que os dados utilizados fornecem bons resultados e auxiliam com boa confiança a identificação dos objetos presentes na planície.

O processo de integração e classificação da imagem SPC-SAR representou avanços importantes para a produção de informações da região amazônica por reduzir as interferências atmosféricas após a união de informações físicoquímicas e morfológicas em um único produto, e permitiu boa delimitação e classificação visual das feições geomorfológicas da área. Este produto foi o que gerou bons resultados de classificação da área, de acordo com os índices de qualidade. Por estes motivos, a integração de dados multisensores é recomendada para aplicação na identificação das feições fluviais da área de estudo. Portanto, a utilização desses sensores permite a realização de análises espaciais e de reconhecimento das feições dos ambientes fluviais com bons resultados e excelente qualidade.

\section{AGRADECIMENTOS}

Os autores agradecem à Pró-Reitoria de Pesquisa, Pós-Graduação e Inovação Tecnológica (Proppit) da Universidade Federal do Oeste do Pará (UFOPA) pela concessão da bolsa de iniciação científica ao primeiro autor e ao Sistema de Proteção da Amazônia (SIVAM) pela disponibilização das imagens SAR (R-99B).

\section{REFERÊNCIAS}

AB'SÁBER, A.N. Zoneamento fisiográfico e ecológico do espaço total da Amazônia Brasileira. Estudos Avançados - São Paulo. v. 24, p. 15-24, 2010.

ARAÚJO, J.F.V., MONTALVÃO R.M.G.; LIMA M.I.C.;

FERNANDES, P.E.C.A.; CUNHA. F.M.B.; FERNANDES,
C.A.C.; BASEI M.A.S. (Coords). Projeto RADAM Brasil. Folha AS-21 SANTARÉM: Geologia, Geomorfologia, Solos, Vegetação e uso potencial da terra. Departamento Nacional de Produção Mineral-Levantamento de Recursos Naturais, Rio de Janeiro. 1976. 
BRYAN, M.L. Use of radar imagery for surface water investigations. In: Satellite Hydrology - Annual William T. Pecora Memorial Symposium. Minneapolis. p. 238-251, 1981.

CHAVEZ, P.S. An improved dark-object subtraction technique for atmospheric scattering correction of multispectral data. Remote Sensing of Environment. v. 24, p. 450-479, 1988.

COHEN, J. A coefficient of agreement for nominal scales. Educ Psychol Meas. v. 46, p. 20-37, 1960.

CUNHA, P.R.C.; GONZAGA, F.G.; COUTINHO, L.F.C.; FEIJÓ, F.J. Bacia do Amazonas. Boletim de Geociências da PETROBRAS. v. 8, p. 47-55, 1994.

GONÇALVES, F.D.; SOUZA FILHO, P.W.M.; PARADELLA, W.R.; MIRANDA, F.P. Fusão de Dados Multisensor para a Identificação e o Mapeamento de Ambientes Flúvio-Estuarinos da Amazônia. Revista Brasileira de Geofísica. v. 27, p. 57-67, 2009.

HARRIS, J.R.; BOWIE, C.; RENEZ, A.N.; GRAHAM, A. Computer-enhancement techniques for integration of remotely sensed, geophysical and thematic data for geosciences. Canadian Journal of Remote Sensing. v. 20, p. 210-221, 1994.

HENDERSON, F.M. \& LEWIS, A.J. Principles \& Applications of Imagining Radar: Manual of Remote Sensing. $3^{\mathrm{a}}$ ed. New York. p. 131-180, 1998.

JENSEN, J.R (Coord.). Introductory digital image processing - A remote sensing perspective. Prentice Hall. 318 p., 1996.

LEHNER, B. \& DOLL, P. Development and validation of a global database of lakes, reservoirs and wetlands. Journal of Hydrology. v. 296, p. 1-22, 2004.

LEWIN, J. Floodplain construction and erosion. In: PETTOS, G. \& CALOW, P. (Coordenadores), River Flows and Channel Forms. Blackwell Science, p. 220, 1996.

MELACK, J.M.; HESS, L.L.; SIPPEL, S. Remote Sensing of lakes and floodplains in the Amazon Basin. Remote Sensing Review. v. 10, p. 127-142, 1994.

NOVO, E.M.L.M.; SHIMABUKURO, Y.E.; MERTES, L. O rio Amazonas em mosaico. Ciência Hoje. v. 24, n. 144, p. 59-61, 1998.

PACHECO, J.B.; BRANDÃO, J.C.M. Geomorfologia fluvial do rio Solimões/Amazonas: estratégias do povo varzeano do Sudoeste do careiro da várzea. Revista Geonorte, v. 2, p. 542554, 2012.
PARADELLA, W.R.; SANTOS, A.R.; VENEZIANI, P.; MORAES, M.C. Synthetic aperture radar for Geological applications in the moist tropics: experiences from the Brazilian Amazon region. Revista Brasileira de Geociências, v. 30, p. 538-542, 2000.

POHL, C. Tools and methods used in data fusion. In: GUNDMANDSEN, P. (Ed). Future trends in Remote Sensing. Rotterdam. v. 10. p. 391-399, 1998.

RODRIGUES, S.W.P. \& SOUZA FILHO, P.W.M. Use of MultiSensor Data to Identify and Map Tropical Coastal Wetlands in the Amazon of Northern Brazil. Wetlands. v. 31, p. 11-23, 2011.

SILVA, T.S.F.; COSTA, M.P.F.; MELACK, J.; NOVO, E.M.L. M. Influência de variações na intensidade do pulso de inundação sobre a cobertura e crescimento da vegetação herbácea aquática na Amazônia. In: SIMPÓSIO BRASILEIRO DE SENSORIAMENTO REMOTO, 2011, Curitiba. Trabalhos Completos...Curitiba: INPE, 2011, v. 15, p. 3205 3212.

TEIXEIRA, S.G. Análise de imagens de sensores remotos orbitais para mapeamento de ambientes costeiros Tropicais e de índices de sensibilidade ambiental ao derramamento de óleo no Golfão Maranhense. Belém, Pará, 2006. 93 p. Dissertação (Mestrado em Geologia e Geoquímica), Instituto de Geociências, Universidade Federal do Pará.

WALD, L. An Overview of concepts in fusion of Earth data. In: GUNDMANDSEN, P. (Ed). Future trends in Remote Sensing. Rotterdam. v. 10. p. 385-390, 1998.

WITTMANN, F.; JUNK, W.J.; PIEDADE, M.T. F. The Varzea forests in Amazonia: flooding and the highly dynamic geomorphology interact with natural forest succession. Forest ecology and Management. v. 196, p. 199-21, 2004.

Submetido em 25 de agosto de 2018 Aceito para publicação em 5 de junho de 2020 